\title{
Hypoxic Non-Small-Cell Lung Cancer Cell-Secreted Exosomal microRNA-582-3p Drives Cancer Cell Malignant Phenotypes by Targeting Secreted Frizzled-Related Protein I
}

This article was published in the following Dove Press journal:

Cancer Management and Research

\author{
Jian Wang \\ Jia Zhao ${ }^{2}$ \\ Jinsong $Z \mathrm{hu}^{\prime}$ \\ Shengli Zhang' \\ 'Department of Respiration, People's \\ Hospital of Cangzhou, Cangzhou, Hebei, \\ People's Republic of China; ${ }^{2}$ Department \\ of Neonatology, People's Hospital of \\ Cangzhou, Cangzhou, Hebei, People's \\ Republic of China
}

Background: Hypoxic environment and exosomes (exos)-mediated intercellular communication are crucial for cancer invasion and metastasis, but the mechanisms are not yet fully understood. In this study, we investigated the regulatory effect of hypoxic tumor cell-secreted exosomal miR-582-3p on non-small-cell lung cancer (NSCLC) cell malignant phenotypes.

Methods: The concentration and diameters of exos were evaluated by nanosight particle tracking analysis. microRNA-582-3p (miR-582-3p) expression was detected by quantitative real-time PCR. The fluorescent dye PKH26 was used to label exos. The direct interaction between miR-582-3p and secreted frizzled-related protein 1 (SFRP1) was determined by dual-luciferase activity assay. NSCLC cell proliferation, migration, and invasion abilities were assessed by cell count kit- 8 assay, wound healing assay, and transwell migration and invasion assay. Western blot analysis was performed to detect the protein expression level.

Results: Hypoxic NSCLC cell-derived exos promoted the proliferation, migration, and invasion of normoxic NSCLC cells. miR-582-3p expression was upregulated in hypoxic NSCLC cells and hypoxic NSCLC cell-secreted exos. Hypoxic NSCLC cell-derived exos transmitted miR-582-3p to normoxic NSCLC cells. Hypoxic NSCLC cell-secreted exosomal miR-582-3p promoted the proliferation, migration, and invasion of normoxic NSCLC cells. miR-582-3p inhibited the expression of SFRP1 protein by binding to its 3'-UTR. In addition, enforced expression of SFRP1 restrained malignant phenotypes of normoxic NSCLC cells, which was abrogated by hypoxic NSCLC cell-secreted exosomal miR-582-3p.

Conclusion: Hypoxic NSCLC cell-secreted exosomal miR-582-3p drives cancer cell malignant phenotypes by targeting SFRP1, which provides a better understanding of cancer metastasis and may facilitate the development of therapeutics against human NSCLC.

Keywords: hypoxia, non-small-cell lung cancer, exosome, microRNA-582-3p, secreted frizzled-related protein 1

\section{Introduction}

Lung cancer is one of the most common malignancies throughout the world, of which non-small-cell lung cancer (NSCLC) is the most primary type in the clinic. ${ }^{1}$ NSCLC is considered as the major leading cause of cancer-related mortality. ${ }^{2}$ Despite great progress has been achieved in the last years, however, the prognosis of patients with NSCLC is still disappointed, with a 5-year survival of about $4 \%{ }^{3}$ Hence, developing a novel and effective therapeutic method is the key to treat NSCLC.
Correspondence: Jian Wang Department of Respiratory, People's Hospital of Cangzhou, 7 Qingchi Avenue, Cangzhou 061000, Hebei, People's Republic of China

Email jianw205@I26.com 
Hypoxia is a basic characteristic of the tumor microenvironment, which is triggered by the rapid expansion and irregular blood flow. ${ }^{4}$ Hypoxia was proved to has a powerful impact on tumor progression, including tumor differentiation, growth, and angiogenesis. ${ }^{5}$ It was discovered that multiple mechanisms, such as high metabolism and oxygen consumption, lead to tumor cell hypoxia, which in turn activates the hypoxia-inducible factor signaling pathway and thereby promotes the proliferation and migration of tumor cells. ${ }^{6}$ Exosomes (exos), small extracellular vesicles with a diameter of 30-200 nm, are shed from the cell membrane into the extracellular matrix. ${ }^{7}$ Exos contain various biologically active molecules, such as proteins, RNAs and lipids, and then take part in a variety of cellular processes through transmitting these molecules from cell-to-cell, so it is also called "the intercellular messenger". 89 There is mounting evidence that exos that derived from tumor cells participate in the regulation of tumor growth and metastasis. ${ }^{10}$ However, the functional role of hypoxic NSCLC cell-secreted exos in NSCLC progression remains poorly understood.

microRNAs (miRNAs) are a class of non-coding oligonucleotides with about $22 \mathrm{nt}$ in length, which serve as essential players in biological processes, including tumorigenesis. ${ }^{11}$ Several lines of evidence have shown that exosomal miRNA can be transmitted to the recipient cells, thereby influencing the function of recipient cells. ${ }^{12}$ As an example, exosomal miR-96 from H1299 cells facilitated the progression of NSCLC by suppressing the expression of LIM-domain only protein $7 .{ }^{13} \mathrm{miR}-582-3 \mathrm{p}$, a novel discovered miRNA, has been reported to play a vital role in tumorigenesis. A previous study has documented that miR-582-3p was downregulated in leukemia cells and its overexpression impaired the proliferation of leukemia cells by inhibiting the expression of cyclin B2. ${ }^{14}$ miR-582-3p has been reported to be upregulated in NSCLC cell lines and tissues, and it could promote tumorigenesis and tumour recurrence. ${ }^{15}$. However, whether hypoxic NSCLC cell-secreted exosomal miR-582-3p participates in the progression of NSCLC has never been extensively investigated.

In this research, we sought to explore the contribution of hypoxic NSCLC cell-secreted exosomal miR-582-3p on NSCLC cell malignant phenotypes. Our findings revealed that hypoxic NSCLC cell-secreted exosomal miR-582-3p, by targeting secreted frizzled-related protein 1 (SFRP1), promoted the malignant phenotypes of NSCLC. Our data provide a novel promising therapeutic approach for NSCLC.

\section{Materials and Methods Cell Culture}

Two NSCLC cell lines (A549 and H1299) acquired from the American Type Culture Collection (ATCC; Manassas, VA, USA) were maintained in Dulbecco's Modified Eagle's Medium (DMEM; Gibco) containing 10\% fetal bovine serum (FBS; Gibco), $100 \mathrm{U} / \mathrm{mL}$ of penicillin, and $100 \mu \mathrm{g} /$ $\mathrm{mL}$ of streptomycin in a humidified incubator containing $95 \%$ air atmosphere and $5 \% \mathrm{CO}_{2}$ at $37^{\circ} \mathrm{C}$.

\section{Tissue Samples}

NSCLC tissues and adjacent non-tumor tissues were obtained from NSCLC patients who underwent surgical resection before radiotherapy or chemotherapy in the People's hospital of Cangzhou. All the NSCLC patients were diagnosed by histopathological examination. Collection of tissue samples was performed with an informed consent signed by each patient. Tissue samples were quickly frozen with liquid nitrogen after excision, and then stored at $-80^{\circ} \mathrm{C}$. This study has been reviewed and approved by the Ethics Committee of the People's hospital of Cangzhou.

\section{Cell Transfection}

SFRP1 overexpression vector (SFRP1-oe.) was established by introducing the full sequence of SFRP1 to the pcDNA 3.0 vector. miR-582-3p mimics and miR-NC were obtained from Sangon (Shanghai, China). Cell transfection was untaken using Lipofectamine ${ }^{\mathrm{TM}} 2000$ Transfection Reagent (Thermo Fisher Scientific, San Jose, CA, USA) according to the manufacturer's instruction.

\section{Isolation of Exos}

A549 and $\mathrm{H} 1299$ cells were cultured for $24 \mathrm{~h}$ under hypoxic $\left(1 \% \mathrm{O}_{2}\right)$ or normoxic $\left(95 \%\right.$ air atmosphere and $\left.5 \% \mathrm{CO}_{2}\right)$ conditions in Dulbecco's modified Eagle's medium (DMEM) supplemented with $10 \%$ exo-free fetal bovine serum (Life Technologies). Exos in the culture medium or serum samples from NSCLC patients or healthy donors were extracted using an exosome purification kit (Norgen Biotek, Thorold, ON, Canada) as per the manufacturer's instruction.

\section{Nanosight Particle Tracking Analysis}

Nanosight particle tracking analysis was used to detect the concentration and size distribution of exos using the Nanosight LM10 system (Nanosight, Amesbury, UK. The exos were resuspended in PBS solution and then injected into the Nanosight LM10 instrument. The capture settings 
and analysis settings were undertaken following the instructions. The exos were analyzed using the NTA analytical software.

\section{Western Blot Analysis}

Proteins were isolated and resolved by SDS-PAGE, and then transferred to PVDF membranes. The PVDF membrane was blocked for $1 \mathrm{~h}$ in $5 \%$ non-fat milk dissolved in tris-buffered saline-Tween 20. The PVDF membrane was incubated with anti-SFRP1 (Abcam, Cambridge, MA, USA) or antiGAPDH antibodies (Abcam) at $4{ }^{\circ} \mathrm{C}$ overnight. After washing, the PVDF membrane was incubated with a secondary HRP-conjugated antibody for $1 \mathrm{~h}$ at $25^{\circ} \mathrm{C}$. Immunoreactive bands were visualized with enhanced chemiluminescence detection reagents (Beyotime, Shanghai, China). The experiment was carried out at least three times.

\section{Quantitative Real-Time PCR (qRT-PCR)}

Total RNA was isolated from cultured NSCLC cells or exos (total protein concentration: approximately $800 \mu \mathrm{g} / \mathrm{mL}$ ) with TRIzol reagent (Thermo Fisher Scientific), and then reverse transcribed to cDNA using a reverse transcription kit (Vazyme, Nanjing, China). Detection of miR-582-3p expression was performed with miRNA Universal SYBR qPCR Master Mix (Vazyme) following protocols supplied by the manufacturer. U6 was used as the internal control to normalize miR-582-3p expression in cells. cel-miR-39 was used to normalize miR-582-3p expression in exos. The sequences of primers were listed as follows: miR-582-3p, forward: $5^{\prime}-\mathrm{G}$ CACACATTGAAGAGGACAGAC-3', reverse: 5'-TATTG AAGGGGGTTCTGGTG-3', U6, forward: 5'-CTCGCTT CGGCAGCACA-3', reverse: 5'-AACGCTTCACGAATTT GCGT-3'. cel-miR-39, forward: 5'-ACACTCCAGCTGGGT CACCGGGTGTAAATC-3'， reverse: 5'-TGGTGTCGTG GAGTCG-3'. The $2^{-\Delta \Delta C t}$ method was applied to analyze relative miR-582-3p expression level in cells. For the normalization of miR-582-3p in exos, $5 \mathrm{nM}$ cel-miR-39 (Qiagen, Hilden, Germany) was added in the isolated RNA sample during reverse transcription. The expression level of miR-582-3p in exos was analyze based on $\mathrm{Ct}$ values, and normalized by cel-miR-39, which was present in each reaction mixture.

\section{Cell Counting Kit-8 (CCK-8) Assay}

NSCLC cells were planted in the 96-well plate $\left(5 \times 10^{3}\right.$ cells per well) in triplicate. After culture for $24 \mathrm{~h}$, NSCLC cells were transfected with empty vector or SFRP1-oe. using Lipofectamine 2000 Transfection Reagent
(Thermo Fisher Scientific) following protocols supplied by the manufacturer, and then treated with miR-582-3pexos $(10 \mu \mathrm{g} / \mathrm{mL})$, followed by incubation for indicated times (12 h, 24 h, 48 h). Subsequently, $10 \mu \mathrm{L}$ CCK-8 solution (Beyotime) was added to each well of the 96well plate. After incubation for $2 \mathrm{~h}$, the absorbance values was detected at $450 \mathrm{~nm}$.

\section{Wound Healing Assay}

NSCLC cells $\left(5 \times 10^{5} /\right.$ well $)$ were plated in a 6 -well plate and incubated overnight in DMEM medium without serum. A sterile $200 \mu \mathrm{L}$ pipette tip was used to create a wound on the monolayer of cells and the floating cells were washed away with PBS. Then the cells were cultured in the medium containing exos $(10 \mu \mathrm{g} / \mathrm{mL})$ for $24 \mathrm{~h}$. At 0 $\mathrm{h}$ and $24 \mathrm{~h}$, the wound was photographed using an optical microscope (Nikon, Japan), and analyzed with the Image $\mathrm{J}$ (NIH, USA). Each experiment was repeated at least 3 times.

\section{Transwell Assay}

The upper chamber was coated with matrigel (Corning, NY, USA) or not to test the ability of cell invasion and migration, respectively. Serum-free DMEM containing (invasion: $2.5 \times 10^{4}$ cells; migration: $1 \times 10^{4}$ cells) was added to the top of the chamber. The lower chamber contains DMEM supplement with 10\% FBS and exos (10 $\mu \mathrm{g} / \mathrm{mL})$. After incubation for $24 \mathrm{~h}$, the residual cells on the upper chamber were removed by a cotton swab. The cells passed through the membrane to the lower surface were fixed with $4 \%$ paraformaldehyde and then stained with $0.5 \%$ crystal violet. The migrative and invasive cells were photographed and counted from 5 randomly selected fields using an optical microscope (Nikon, Japan). The experiment was carried out at least three times.

\section{Dual-Luciferase Activity Assay}

The wild-type (WT) or mutant (MUT) 3'UTR fragment of SFRP1 mRNA containing a putative miR-582-3p-binding site was subcloned into the pGL3 vector (Promega Corporation) to construct SFRP1 3'UTR-WT or SFRP1 3'UTR-MUT recombinant luciferase plasmids. For the luciferase assay, NSCLC cells were seeded in 24-well plates in triplicate at a concentration of $1 \times 10^{4}$ cells $/ \mathrm{mL}$ and grown overnight. NSCLC cells were co-transfected with SFRP1 3'UTR-WT or SFRP1 3'UTR-MUT luciferase plasmids and miR-582-3p mimics or miR-NC with 
Lipofectamine $^{\mathrm{TM}} 2000$ Transfection Reagent (Thermo

Fisher Scientific) according to the manufacturer's instruction, or treated with A549 cell-derived exos $(10 \mu \mathrm{g} / \mathrm{mL})$. At $48 \mathrm{~h}$ after treatment, luciferase activity was detected with a Dual Luciferase Reporter Assay Kit (Promega) following protocols supplied by the manufacturer.

\section{Statistical Analysis}

All data analysis is performed with SPSS 20.0 software (SPSS Inc., Chicago, IL, USA). Data form three dependent repeat experiments were shown as the means. Statistical analysis is performed using Student's $t$-test or one-way analysis of variance (ANOVA). Statistics with $P$-value $<0.05$ is considered statistically significant.

\section{Results}

Hypoxic NSCLC Cell-Derived Exos

Promote the Proliferation, Migration, and Invasion of Normoxic NSCLC Cells

Exos derived from NSCLC cells were isolated from conditioned medium of normoxic or hypoxic A549 cells. To confirm whether exos were successfully isolated from tumor cells, Western blot assay and nanosight particle tracking analysis were performed. As shown in Figure 1A, exo markers CD63, CD9 and Alix were enriched in exos derived from normoxic or hypoxic A549 cells (Norm-exos and Hypo-exos). Nanosight particle tracking analysis showed that the diameter of particles was about $50-200 \mathrm{~nm}$, indicating that exos were
A

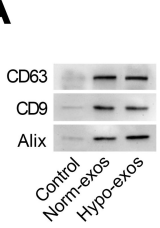

B

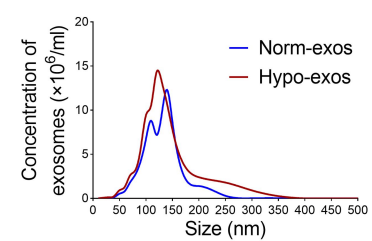

C

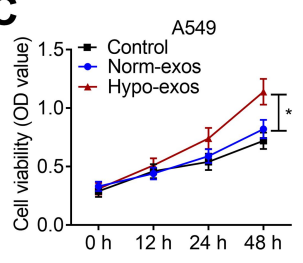

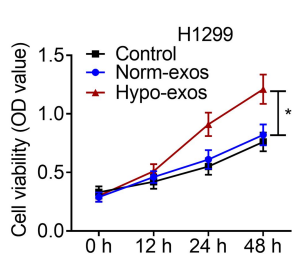

D
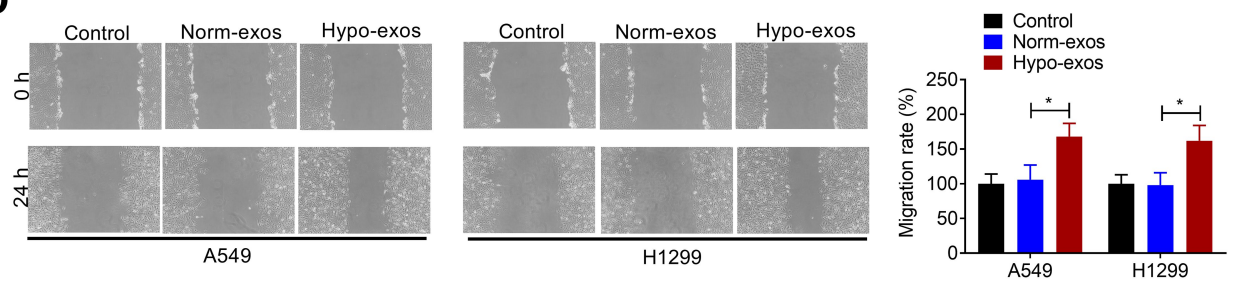

E
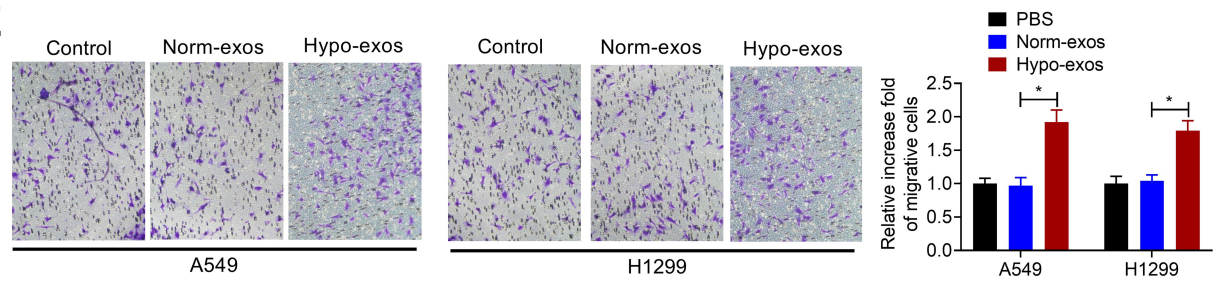

$\mathbf{F}$
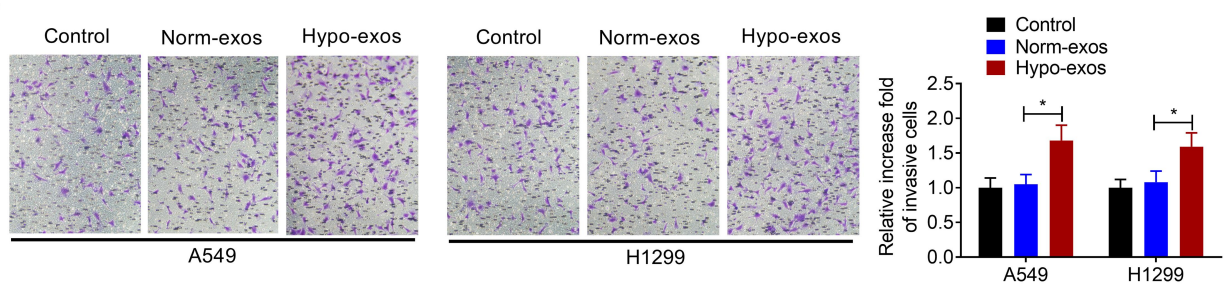

Figure I Hypoxic NSCLC cell-derived exos promote proliferation, migration, and invasion of normoxic NSCLC cells. (A) Western blot analysis of the enrichment of CD63, CD9 and Alix in exos purified from A549 cell culture medium under normoxic (Norm-exos) or hypoxic (Hypo-exos) condition. Control: the medium from A549 cells under normoxic condition. (B) The concentration and size distribution of exos were detected by Nanosight particle tracking analysis. (C) The effect of Norm-exos or Hypo-exos on the viability of NSCLC cell lines A549 and HI299 cells was evaluated by CCK-8 assay. (D and E) The effect of Norm-exos or Hypo-exos on the migration abilities of NSCLC cell lines A549 and HI299 cells was analyzed by wound healing and transwell migration assay. (F) The effect of Norm-exos or Hypo-exos on the invasion abilities of NSCLC cell lines A549 and HI299 cells was analyzed by transwell invasion assay. The experiments were repeated three times. Data were expressed as mean \pm standard deviation. ${ }^{*} \mathrm{p}<0.05$. 
successfully isolated from the medium of A549 cells under normoxic or hypoxic condition (Figure 1B).

Subsequently, we analyzed the effects of Norm-exos and Hypo-exos on the proliferation, migration, and invasion of NSCLC cell lines A549 and H1299. CCK-8 assay showed that Hypo-exos could significantly increase the viability of A549 and H1299 cells compared to the Norm-exos group (Figure 1C). Additionally, the wound healing assay and the transwell migration assay showed that, compared with the Norm-exos group, Hypo-exos treatment could significantly increase the migration abilities of A549 and H1299 cells (Figure 1D and E). Similarly, Hypo-exos also promoted the invasion of A549 and H1299 cells, as shown by transwell invasion assay (Figure 1F). These data suggested that exos derived from hypoxic A549 cells increased the malignant phenotype of normoxic NSCLC cells.

\section{Hypoxia Promotes miR-582-3p Expression in NSCLC Cells and NSCLC Cell-Secreted Exos}

To investigate the impact of hypoxia on the expression of miR582-3p, the levels of miR-582-3p in NSCLC cell lines A549 and H1299 were determined by qRT-PCR assay. The results showed that the level of miR-582-3p in hypoxic NSCLC cells

was higher than that in normoxic NSCLC cells (Figure 2A). In addition, the level of miR-582-3p in exos derived from hypoxic NSCLC cells was also significantly higher than that in exos derived from normoxic NSCLC cells (Figure 2B). Moreover, miR-582-3p level was detected in adjacent normal tissues and NSCLC tissues, and it was found that miR-528-3p was upregulated in NSCLC tissues relative to their adjacent normal tissues (Figure 2C). Exos were isolated from the serum of healthy donors and NSCLC patients, respectively. The results of qRT-PCR assay showed that the level of miR-582$3 p$ in exos derived from the serum of NSCLC patients was significantly higher than that in exos derived from the serum of healthy controls (Figure 2D).

\section{Hypoxic NSCLC Cell-Derived Exos Transmit miR-582-3p to Normoxic NSCLC Cells}

miR-582-3p mimics or the corresponding negative control (NC) was transfected into A549 cells, followed by hypoxic treatment. As shown in Figure 3A, miR-582-3p mimics transfection successfully increased the level of miR-582-3p in A549 cells. At $48 \mathrm{~h}$ after transfection, hypoxic NSCLC cell-derived exos were isolated and purified. The level of miR-528-3p in miR-582-3p-exos was obviously higher than

A

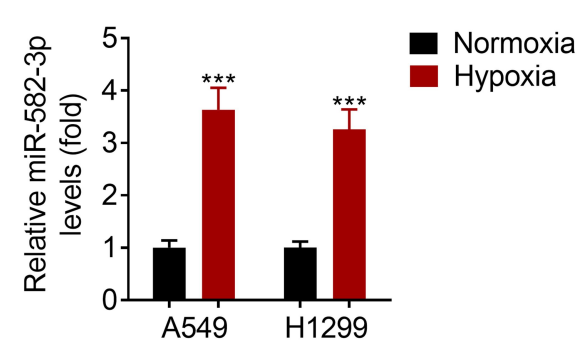

C

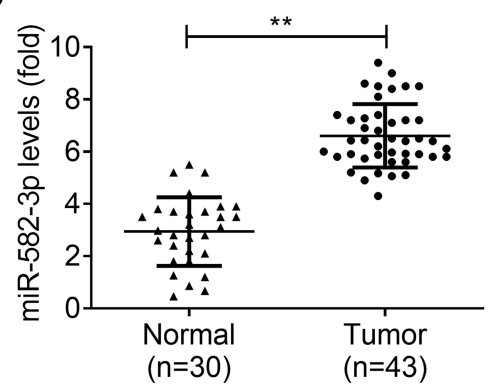

B

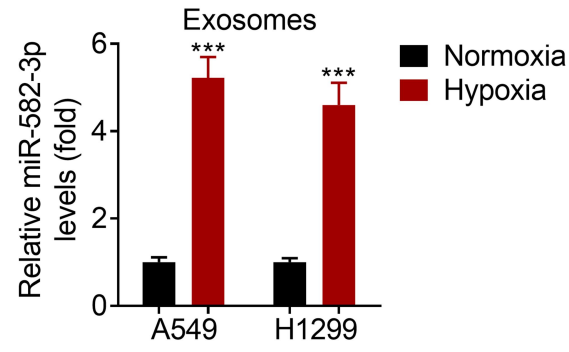

D

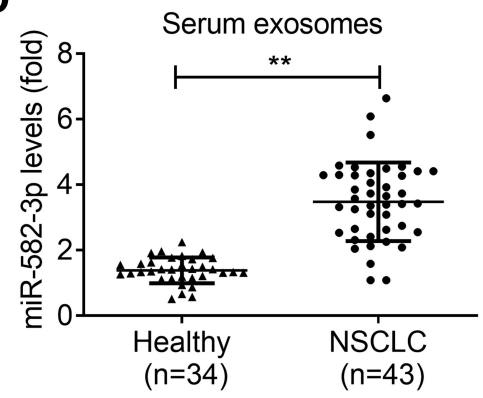

Figure 2 Hypoxia promotes miR-582-3p expression in NSCLC cells and NSCLC cell-secreted exos. (A) The levels of miR-582-3p in hypoxic and normoxic NSCLC cells was detected using qRT-PCR assay. (B) The levels of miR-582-3p in exos derived from hypoxic NSCLC cells and normoxic NSCLC cells were analyzed by qRT-PCR assay. (C) The levels of miR-582-3p in adjacent normal tissues $(n=30)$ and NSCLC tissues $(n=43)$ were analyzed by $q R T-P C R$ assay. $(D)$ Levels of miR-582-3p in serum exos from healthy donors $(n=34)$ and NSCLC patients $(n=43)$ were detected by qRT-PCR assay. The experiments were repeated three times. Data were expressed as mean \pm standard deviation. ${ }^{* *} \mathrm{p}<0.01$ and $* * * \mathrm{p}<0.001$. 
A

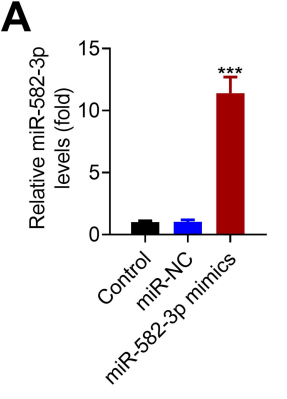

E

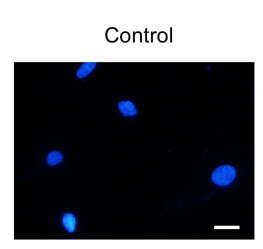

B
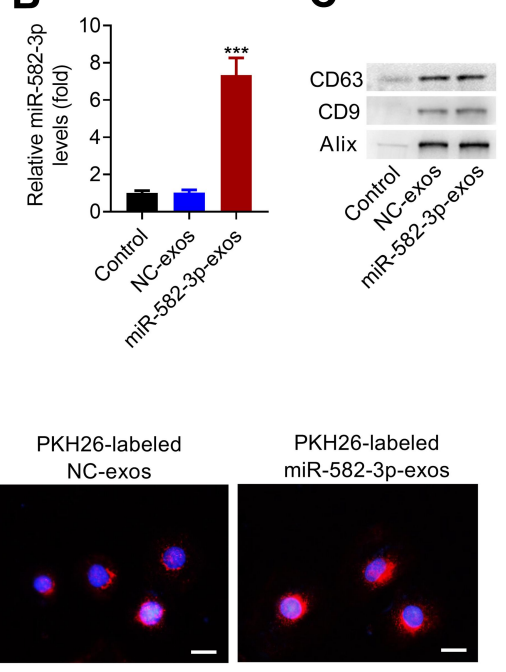

C

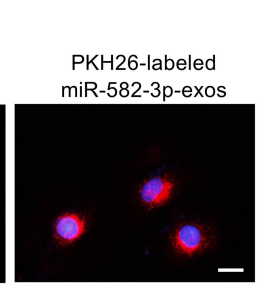

D

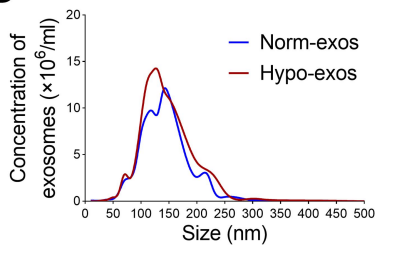

$\mathbf{F}$

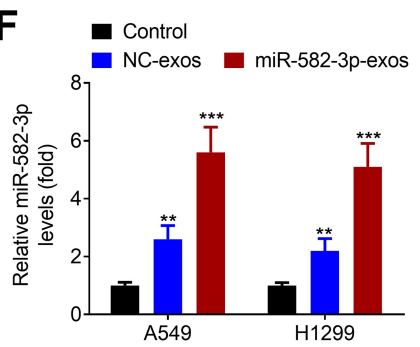

Figure 3 Hypoxic NSCLC cell-derived exos transmit miR-582-3p to normoxic NSCLC cells. (A) miR-582-3p mimics transfection markedly up-regulated the levels of miR582-3p in hypoxic A549 cells. (B) The levels of miR-528-3p in miR-582-3p-exos and NC-exos was analyzed using qRT-PCR assay. (C) Western blot analysis of the enrichment of CD63, CD9 and Alix in exos purified from hypoxic A549 cell line transfected with miR-582-3p mimic (miR-582-3p-exos) or NC (NC-exos). (D) The concentration and size distribution of exos were detected by nanosight particle tracking analysis. (E) The exo uptake assay was conducted to confirm the uptake of PKH26-labeled miR-582-3pexos (red) into recipient normoxic A549 cells. Scale bar: $20 \mu \mathrm{m}$. (F) Both NC-exos or miR-582-3p-exos treatment significantly increased the levels of miR-582-3p in A549 and $\mathrm{HI} 299$ cells. The experiments were repeated three times. Data were expressed as mean \pm standard deviation. $* * \mathrm{p}<0.0 \mathrm{I}$ and $* * * \mathrm{p}<0.00 \mathrm{I}$.

that in NC-exos (Figure 3B). Western blot assay revealed that CD63, CD9 and Alix were enriched in isolated hypoxic A549 cell line-derived exos (Figure 3C). Similarly, nanosight particle tracking analysis also verified the concentration and diameters of exos, indicating that exos were successfully isolated from hypoxic A549 cells transfected with miR528-3p or NC (miR-582-3p-exos or NC-exos; Figure 3D).

Subsequently, we confirmed whether miR-582-3p-exos delivered into normoxic NSCLC cells. The exos were labeled with PKH26 and incubated with A549 cells. As shown in Figure 3E, PKH26 labeled exos entered A549 cells. Then, A549 and H1299 cells were incubated with miR-582-3p-exos or NC-exos. The results of qRT-PCR assay showed that both miR-582-3p-exos and NC-exos significantly increased the levels of miR-582-3p in A549 and H1299 cells compared with the control group (Figure 3F). These results revealed that hypoxic A549 cell-derived exos transmit miR-582-3p to normoxic NSCLC cells.

\section{Hypoxic NSCLC Cell-Secreted Exosomal miR-582-3p Promotes the Proliferation, Migration, and Invasion of Normoxic NSCLC Cells}

To determine whether hypoxic A549 cell-secreted exosomal miR-582-3p affects the biological properties of normoxic
NSCLC cells, A549 and H1299 cells were treated with miR-582-3p-exos or NC-exos. CCK-8 assay showed that NC-exos significantly increased the cell viability of A549 and H1299 cells compared with the control group. Simultaneously, compared with the NC-exos group, the viability of A549 and H1299 cells was significantly increased in the miR-582-3p-exos group (Figure 4A). Wound healing assay and transwell assay also showed that NC-exos treatment promoted the migration and invasion of A549 and H1299 cells. Moreover, the migration and invasion abilities of NSCLC cells in the miR-582-3p-exos group were significantly higher than those in the NC-exos group (Figure 4B and $\mathrm{C}$ ).

\section{SFRPI is a Functional Downstream Target of miR-582-3p}

The predictive binding sites of miR-582-3p on SFRP1 3'UTR was shown (Figure 5A). Western blot assay showed that the level of SFRP1 protein was significantly reduced in A549 cells transfected with miR-582-3p mimic. Similarly, miR-582-3p-exos treatment significantly reduced the protein levels of SFRP1 in A549 cells (Figure 5B and C). To confirm the direct binding between miR-582-3p and SFRP1, we conducted a luciferase reporter assay in A549 and H1299 cells. The luciferase reporter plasmid containing wild-type (WT) or mutant (MUT) SFRP1 mRNA 3'UTR and miR-582-3p 
A

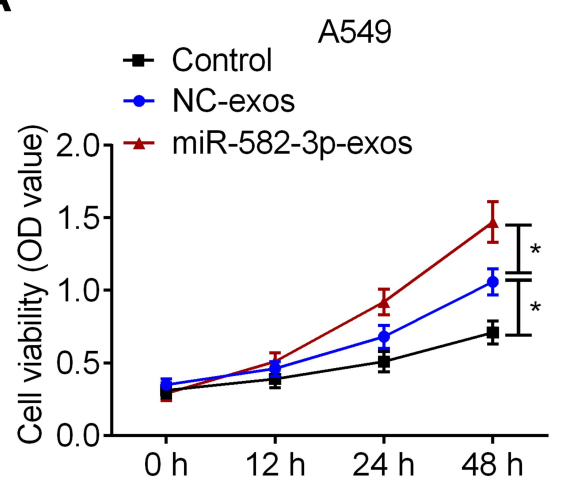

B

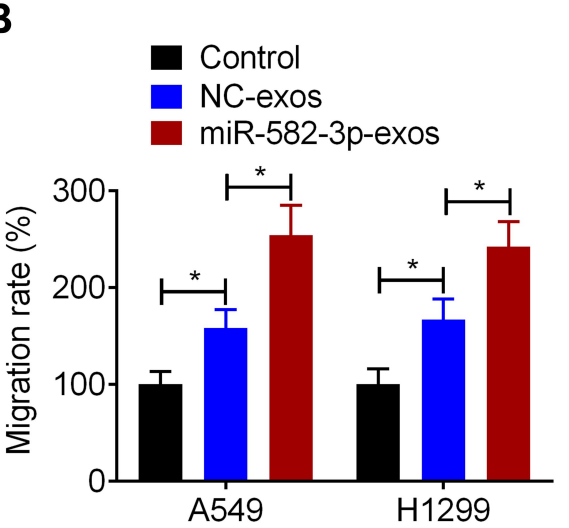

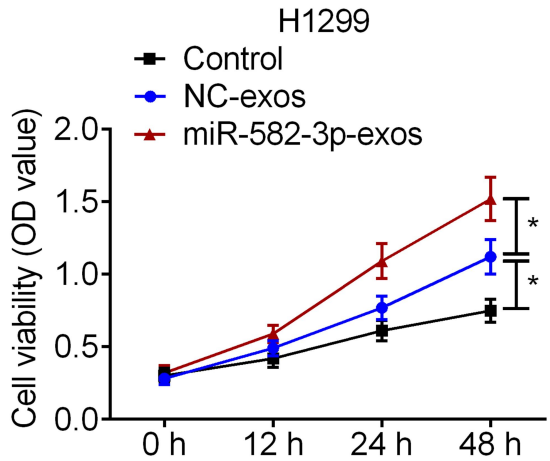

C - Control

- NC-exos

miR-582-3p-exos

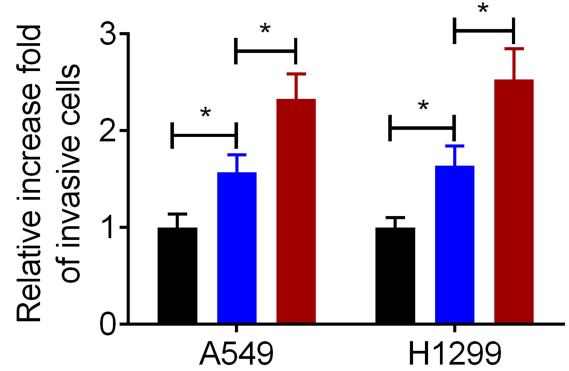

Figure 4 Hypoxic NSCLC cell-secreted exosomal miR-582-3p promotes the proliferation, migration, and invasion of normoxic NSCLC cells. A549 and HI299 cells were incubated with miR-582-3p-exos or NC-exos. (A) The viability of A549 and HI299 cells was detected by CCK-8 assay. The wound healing assay (B) and transwell assay (C) were used to detect the migration and invasion abilities of A549 and HI299 cells. The experiments were repeated three times. Data were expressed as mean \pm standard deviation. ${ }^{*} \mathrm{p}<0.05$.

mimics or miR-NC were co-transfected into A549 and H1299 cells, respectively. The results showed that transfection of miR-582-3p mimics reduced the luciferase activity of SFRP1-WT reporter plasmid compared to the miR-NC group, but did not affect the luciferase activity of SFRP1MUT reporter plasmid (Figure 5D and E). Similarly, the luciferase activity of SFRP1-WT reporter was also reduced by miR-582-3p-exos and NC-exos treatment, while the luciferase activity of SFRP1-MUT reporter was unaffected by miR-582-3p-exos and NC-exos treatment (Figure 5F and G). These findings indicated that SFRP1 was a target of miR582-3p, and miR-582-3p-exos inhibited SFRP1 expression in normoxic NSCLC cells.

\section{miR-582-3p Abrogates the Effect of SFRPI Overexpression on the Malignant Phenotypes of Normoxic NSCLC Cells}

Next, we investigated whether hypoxic A549 cell-secreted exosomal miR-582-3p drives cancer cell malignant phenotypes by targeting SFRP1. A549 and H1299 cells were transfected with SFRP1-oe.or Vector, followed by incubation with miR-582-3p-exos. Western blot assay showed that SFRP1-oe. transfection markedly increased the protein levels of SFRP1 in A549 and H1299 cells, while this effect was suppressed following miR-582-3pexos treatment (Figure 6A). Additionally, CCK-8 assay showed that overexpression of SFRP1 reduced the viability of A549 and H1299 cells, which was blocked after miR582-3p treatment (Figure 6B). In parallel, upregulation of SERP1 enhanced the migration, and invasion of. Whereas, these changes induced by SERP1 overexpression were eliminated by miR-582-3p-exos administration (Figure 6C and D). These preliminary data indicated that hypoxic A549 cell-derived exosomal miR-582-3p enhanced the malignant phenotype of NSCLC cells through targeting SFRP1.

\section{Discussion}

Hypoxia acts as a principal contributor to the progression of human cancers. ${ }^{10}$ Hypoxia causes the dysregulation of 
A

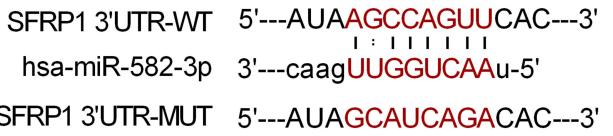

B

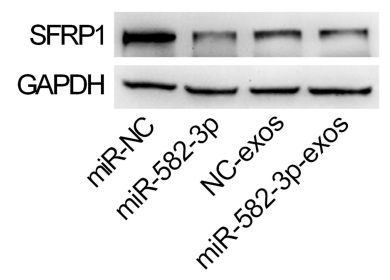

D

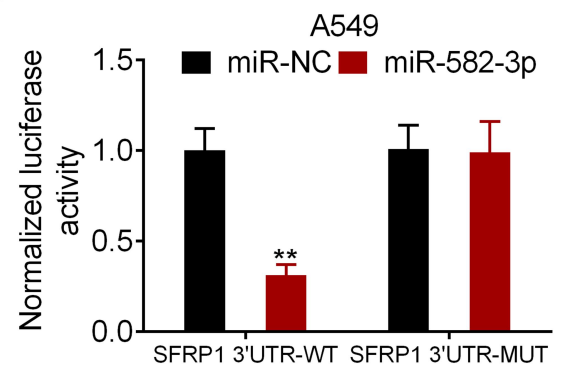

F

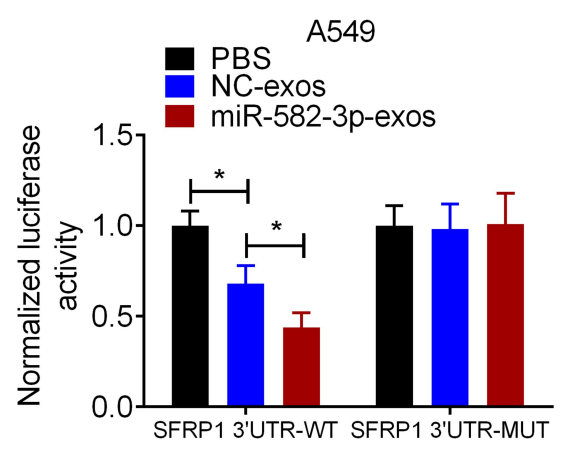

C

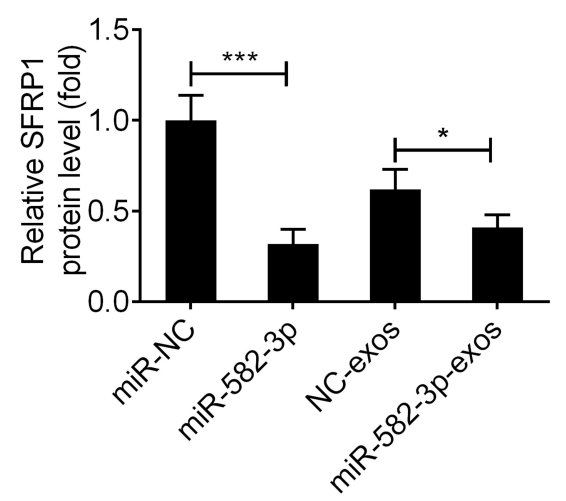

E

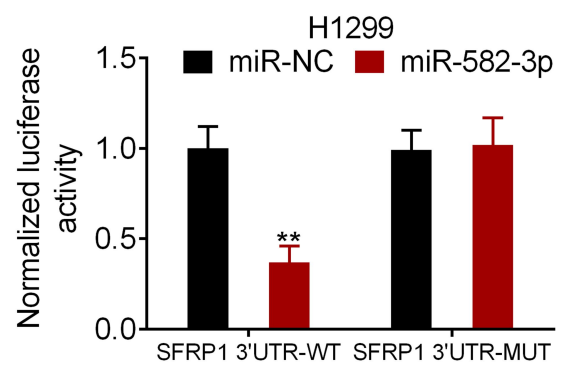

G

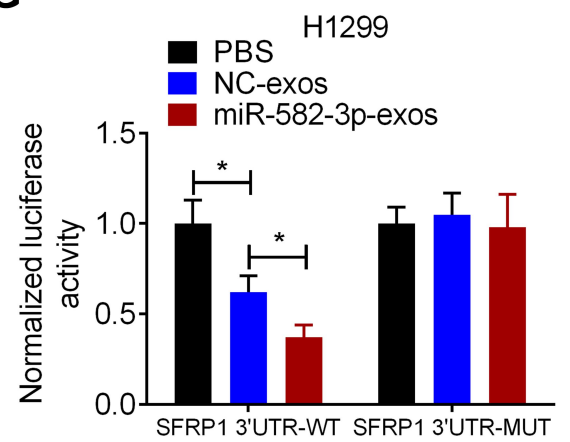

Figure 5 SFRPI is a functional downstream target of miR-582-3p. (A) Prediction binding sites of miR-582-3p in the 3'UTR sequence of SFRPI. (B and C) miR-582-3p mimics or miR-582-3p-exos decreased the expression of SFRPI protein in normoxic A549 cells. (D and E) Luciferase activity in A549 and HI299 cells co-transfected with wild-type (WT) or mutant (MUT) SFRPI 3'UTR reporter and miR-582-3p mimics or miR-NC. (F and G) Luciferase activity of WT or MUT SFRPI 3'UTR reporter in A549 and HI299 cells treated with miR-582-3p-exos or NC-exos. The experiments were repeated three times. Data were expressed as mean \pm standard deviation. $* \mathrm{p}<0.05$, $* * p<0.0 \mathrm{I}$, and $* * * \mathrm{p}<0.001$.

genes involved in various cellular and physiological functions, triggers angiogenesis, and induces tumor cells escaping from the cytotoxic effect of chemotherapeutic agents, ultimately resulting in a more aggressive and lower chemotherapy-sensitive tumor phenotype. ${ }^{16-18}$ It follows that a thorough elucidation of hypoxia-mediated tumorigenesis will provide a novel sight for NSCLC treatment. Previous studies have demonstrated that hypoxic cell-derived exos have a role in the development of human cancer. ${ }^{19}$ Nevertheless, the impact of hypoxic NSCLC cell-secreted exos on the progression of normoxic NSCLC cells has not been poorly studied. In the present report, we found that hypoxic NSCLC cell-secreted exos could enhance the proliferation, migration, and invasion of A549 and H1299 cells under a normoxic condition, further fitting the established notion of hypoxic cell-derived exos as a vital contributor to the development of human cancer.

In the last years, cancer-derived exos have captured extensive attention in tumor research due to their roles in aspects of the transportation of intercellular bioactive molecules. ${ }^{20}$ Previous studies showed that tumor cells could generate more exos than normal cells. Exos derived 
$\mathbf{A}$
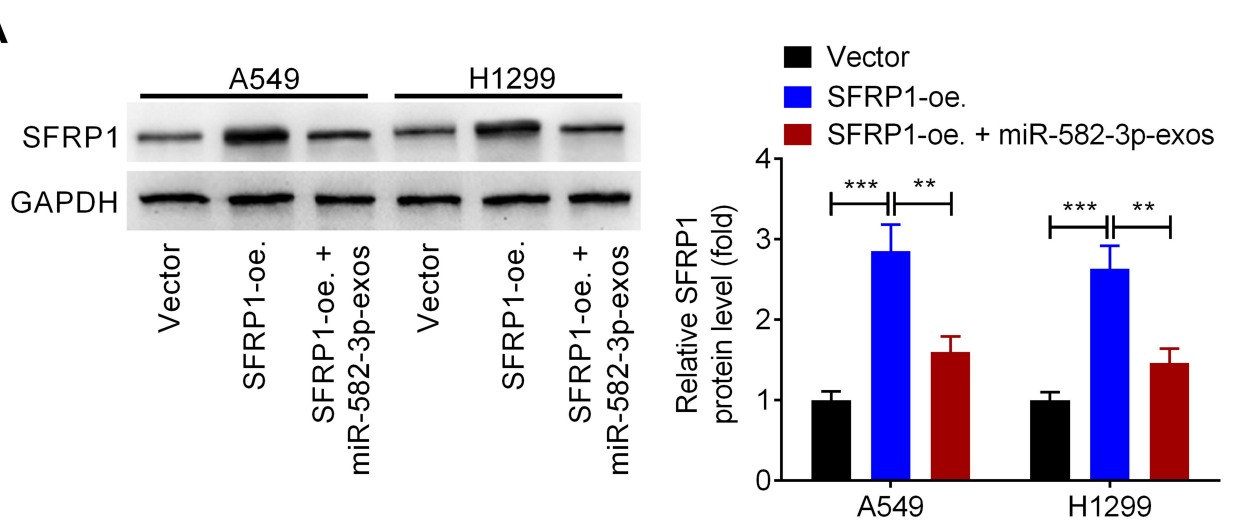

B
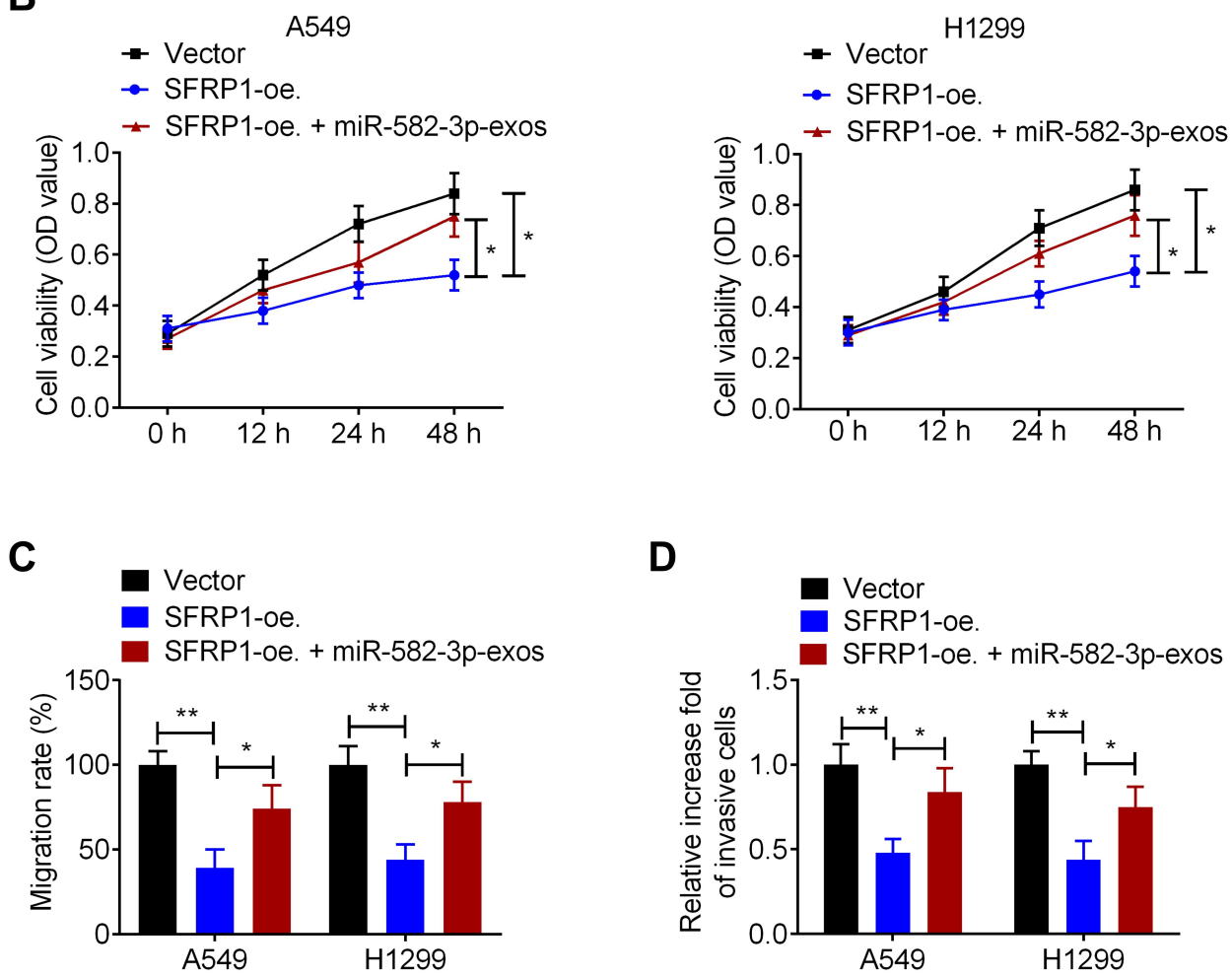

Figure 6 Hypoxic NSCLC cell-secreted exosomal miR-582-3p abrogates the effect of SFRPI on the malignant phenotypes of normoxic NSCLC cells. A549 and HI299 cells were transfected with SFRPI-oe. and then treated with miR-582-3p-exos. (A) The protein levels of SFRPI were analyzed using Western blot assay. (B) The viability of A549 and $\mathrm{HI} 299$ cells was analyzed by CCK-8 assay. The migration and invasion abilities of A549 and HI299 cells were analyzed using wound healing assay (C) and transwell assay (D). The experiments were repeated three times. Data were expressed as mean \pm standard deviation. ${ }^{*} p<0.05$, ${ }^{* *} p<0.01$, and ${ }^{* * *} p<0.001$.

from tumor cells participated in the regulation of cellular functions via delivery of bioactive molecules, such as miRNA. ${ }^{21}$ Notably, the link between cancer cell-secreted exosomal miRNAs and the microenvironment of human cancer has been investigated. It has been demonstrated that cancer-secreted exosomal miRNAs could be horizontally transferred to the target cells, thereby controlling the biology of cancer cells. ${ }^{22}$ For example, exosomal miR-208a from A549 cells, through targeting p21, restrained the proliferation of NSCLC cells via activating the AKT/mechanistic target of rapamycin signaling. ${ }^{23}$ Similarly, exos derived from the gemcitabine-resistant A549 cells transferred miR-222-3p to A549 cells and then promoted the proliferation, migration, and invasion of A549 cells by targeting suppressor of cytokine signaling $3{ }^{24}$ Moreover, hypoxia treatment promoted the production of exos in tumor cells, and exosomal miR-23a secreted by hypoxic lung cancer cells could cause an obvious reduction of tight junction protein ZO-1 expression, thereby facilitating angiogenesis, vascular permeability, and tumor growth in vivo. ${ }^{25}$ Furthermore, exosomal miR-21 derived from hypoxic NSCLC cells inhibited the sensitivity of normoxic NSCLC cells to cisplatin through inhibition of 
phosphatase and tensin homolog. ${ }^{26}$ In this study, we found that hypoxia caused a striking elevation of miR-582-3p expression in NSCLC cells and NSCLC cell-secreted exos. Interestingly, the dysregulation of miR-582-3p was discovered in several types of human cancers, such as colon cancer and non-functioning pituitary adenomas. ${ }^{27,28}$ miR-582-3p was reported to be downregulated in prostate cancer tissues and tightly associated with advanced clinicopathological characteristics and poor prognosis in patients with prostate cancer. Moreover, overexpression of miR-582-3p restrained the metastasis of prostate cancer cells in vitro and mitigated bone metastasis in vivo by repressing the transforming growth factor $\beta$ signaling. ${ }^{29}$ Antagonizing miR-582-3p strongly repressed the tumor progression in vivo via activation of the Wnt/ $\beta$-catenin signaling pathway, indicating that miR-582-3p is a potential therapeutic target for NSCLC. ${ }^{15}$ However, whether hypoxic NSCLC cell-derived exosomal miR-582-3p participates in controlling the malignant phenotypic traits of NSCLC cells has never been reported. Herein, miR-582-3p was confirmed to be transferred from hypoxic NSCLC cell-secreted exos to the normoxic NSCLC cells, suggesting that hypoxic NSCLC cell-secreted exosomal miR-582-3p serves as a crucial player in the development of NSCLC. Furthermore, we found that hypoxic NSCLC cell-derived exosomal miR-582-3p could promote the proliferation, migration, and invasion of normoxic NSCLC cells, revealing the tumor-promoting role of hypoxic NSCLC cellderived exosomal miR-582-3p in NSCLC.

There is no denying that miRNAs exert their potent suppressive activities on target genes by binding to the 3'UTR of messenger RNA, thereby potentially controlling a variety of cellular processes. ${ }^{30}$ To further investigate the mechanism underlying hypoxic NSCLC cell-derived exosomal miR-582$3 p$ action on NSCLC progression, we sought to identify the downstream target gene of miR-582-3p and found that there were miR-582-3p-binding sites within the sequence of SFRP1. SFRP1 is a natural inhibitor of canonical Wnt signaling and its aberrant expression has been discovered in various human cancers. ${ }^{31}$ The downregulation of SFRP1 was discovered in hepatoblastoma and its overexpression diminished the proliferation and migration of hepatoblastoma cells. ${ }^{32}$ In addition, in a skin carcinogenesis model, SFRP1 knockout mice showed early tumor initiation and increased tumorigenic potential of cancer stem cells. Moreover, the downregulation of SFRP1 was correlated with poor survival in patients with epithelial cancers, suggesting the involvement of SFRP1 in tumorigenesis. ${ }^{33}$ Whereas, whether the action of hypoxic NSCLC cell-derived exosomal miR-582-3p on NSCLC progression is mediated by SFRP1 has not been studied previously. Herein, the interaction of miR-582-3p and SFRP1 was identified in NSCLC cells. More importantly, we found that enforced expression of SFRP1 suppressed malignant phenotypes of normoxic NSCLC cells, and this action was abolished by hypoxic NSCLC cell-secreted exosomal miR-582-3p, revealing that hypoxic NSCLC cell-derived exosomal miR582-3p enhanced the malignant phenotype of NSCLC cells through targeting SFRP1. As the deficiency of this study, the tumor-promoting activity of hypoxic NSCLC cell-derived exosomal miR-582-3p was not identified in NSCLC xenografted nude mouse models. This is the focus of our future work.

In summary, the tumor-promoting effect of hypoxic NSCLC cell-derived exos on NSCLC progression has been identified in our study. miR-582-3p is highly enriched in exos derived from hypoxic NSCLC cells, and exo-mediated transfer of miR-582-3p could drive cancer cell malignant phenotypes by targeting SFRP1. Our findings contribute to the understanding of the function of exosomal miR-582-3p in NSCLC progression and provide a new sight for the management of patients with NSCLC.

\section{Disclosure}

The authors report no conflicts of interest for this work.

\section{References}

1. Arbour KC, Riely GJ. Systemic therapy for locally advanced and metastatic non-small cell lung cancer: a review. JAMA. 2019;322 (8):764-774. doi:10.1001/jama.2019.11058

2. Fitzmaurice C, Allen C, Barber RM, et al. Global, regional, and national cancer incidence, mortality, years of life lost, years lived with disability, and disability-adjusted life-years for 32 cancer groups, 1990 to 2015: a systematic analysis for the global burden of disease study. JAMA Oncol. 2017;3(4):524-548. doi:10.1001/jamaoncol. 2016.5688

3. Siegel RL, Miller KD, Jemal A. Cancer statistics, 2018. CA Cancer J Clin. 2018;68(1):7-30. doi:10.3322/caac.21442

4. Riera-Domingo C, Audige A, Granja S, et al. Immunity, hypoxia, and metabolism-the menage a trois of cancer: implications for immunotherapy. Physiol Rev. 2020;100(1):1-102. doi:10.1152/ physrev.00018.2019

5. Kakkad S, Krishnamachary B, Jacob D, et al. Molecular and functional imaging insights into the role of hypoxia in cancer aggression. Cancer Metastasis Rev. 2019;38(12):51-64. doi:10.1007/s10555-019-09788-3

6. Macklin PS, McAuliffe J, Pugh CW, et al. Hypoxia and HIF pathway in cancer and the placenta. Placenta. 2017;56:8-13. doi:10.1016/j. placenta.2017.03.010

7. Kalishwaralal K, Kwon WY. Exosomes for non-invasive cancer monitoring. Biotechnol J. 2019;14(1):e1800430. doi:10.1002/ biot. 201800430

8. Milman N, Ginini L, Gil Z. Exosomes and their role in tumorigenesis and anticancer drug resistance. Drug Resist Updat. 2019;45:1-12. doi:10.1016/j.drup.2019.07.003 
9. Wortzel I, Dror S, Kenific CM, et al. Exosome-mediated metastasis: communication from a distance. Dev Cell. 2019;49(3):347-360. doi:10.1016/j.devcel.2019.04.011

10. Graham K, Unger E. Overcoming tumor hypoxia as a barrier to radiotherapy, chemotherapy and immunotherapy in cancer treatment. Int J Nanomed. 2018;13:6049-6058. doi:10.2147/ijn. s140462

11. Takahashi RU, Prieto-Vila M, Kohama I, et al. Development of miRNA-based therapeutic approaches for cancer patients. Cancer Sci. 2019;110(4):1140-1147. doi:10.1111/cas.13965

12. Ghayad SE, Rammal G, Ghamloush F, et al. Exosomes derived from embryonal and alveolar rhabdomyosarcoma carry differential miRNA cargo and promote invasion of recipient fibroblasts. Sci Rep. 2016;6:37088. doi:10.1038/srep37088

13. Wu H, Zhou J, Mei S, et al. Circulating exosomal microRNA-96 promotes cell proliferation, migration and drug resistance by targeting LMO7. J Cell Mol Med. 2017;21(6):1228-1236. doi:10.1111/ jcmm. 13056

14. Li H, Tian X, Wang P, et al. MicroRNA-582-3p negatively regulates cell proliferation and cell cycle progression in acute myeloid leukemia by targeting cyclin B2. Cell Mol Biol Lett. 2019;24:66. doi:10.1186/s11658-019-0184-7

15. Fang L, Cai J, Chen B, et al. Aberrantly expressed miR-582-3p maintains lung cancer stem cell-like traits by activating Wnt/ $\beta$ catenin signalling. Nat Commun. 2015;6:8640. doi:10.1038/ ncomms 9640

16. Roma-Rodrigues C, Mendes R, Baptista PV, et al. Targeting tumor microenvironment for cancer therapy. Int J Mol Sci. 2019;20:4. doi:10.3390/ijms20040840

17. Redfern A, Agarwal V. Hypoxia as a signal for prison breakout in cancer. Curr Opin Clin Nutr Metab Care. 2019;22(4):250-263. doi: $10.1097 / \mathrm{mco} 0000000000000577$

18. Sruthi TV, Edatt L, Raji GR, et al. Horizontal transfer of miR-23a from hypoxic tumor cell colonies can induce angiogenesis. $J$ Cell Physiol. 2018;233(4):3498-3514. doi:10.1002/jcp.26202

19. Shao C, Yang F, Miao S, et al. Role of hypoxia-induced exosomes in tumor biology. Mol Cancer. 2018;17(1):120. doi:10.1186/s12943018-0869-y

20. Blackwell RH, Foreman KE, Gupta GN. The role of cancer-derived exosomes in tumorigenicity \& epithelial-to-mesenchymal transition. Cancers. 2017;9:8. doi:10.3390/cancers9080105

21. Kumar A, Deep G. Hypoxia in tumor microenvironment regulates exosome biogenesis: molecular mechanisms and translational opportunities. Cancer Lett. 2020;479:23-30. doi:10.1016/j.canlet. 2020.03 .017
22. $\mathrm{Hu} \mathrm{C}$, Meiners $\mathrm{S}$, Lukas $\mathrm{C}$, et al. Role of exosomal microRNAs in lung cancer biology and clinical applications. Cell Prolif. 2020: e12828. doi:10.1111/cpr.12828.

23. Tang Y, Cui Y, Li Z, et al. Radiation-induced miR-208a increases the proliferation and radioresistance by targeting p21 in human lung cancer cells. J Exp Clin Cancer Res. 2016;35:7. doi:10.1186/ s13046-016-0285-3

24. Wei F, Ma C, Zhou $\mathrm{T}$, et al. Exosomes derived from gemcitabine-resistant cells transfer malignant phenotypic traits via delivery of miRNA-222-3p. Mol Cancer. 2017;16(1):132. doi:10.1186/s12943-017-0694-8

25. Hsu YL, Hung JY, Chang WA, et al. Hypoxic lung cancer-secreted exosomal miR-23a increased angiogenesis and vascular permeability by targeting prolyl hydroxylase and tight junction protein ZO-1. Oncogene. 2017;36(34):4929-4942. doi:10.1038/onc.2017.105

26. Dong C, Liu X, Wang H, et al. Hypoxic non-small-cell lung cancer cell-derived exosomal miR-21 promotes resistance of normoxic cell to cisplatin. Onco Targets Ther. 2019;12:1947-1956. doi:10.2147/ott. s186922

27. Bobowicz M, Skrzypski M, Czapiewski P, et al. Prognostic value of 5-microRNA based signature in T2-T3N0 colon cancer. Clin Exp Metastasis. 2016;33(8):765-773. doi:10.1007/s10585-016-9810-1

28. Butz H, Liko I, Czirjak S, et al. MicroRNA profile indicates downregulation of the TGFbeta pathway in sporadic non-functioning pituitary adenomas. Pituitary. 2011;14(2):112-124. doi:10.1007/s11102-0100268-x

29. Huang S, Zou C, Tang Y, et al. miR-582-3p and miR-582-5p suppress prostate cancer metastasis to bone by repressing TGF-beta signaling. Mol Ther Nucleic Acids. 2019;16:91-104. doi:10.1016/j. omtn.2019.01.004

30. Rupaimoole R, Slack FJ. MicroRNA therapeutics: towards a new era for the management of cancer and other diseases. Nat Rev Drug Discov. 2017;16(3):203-222. doi:10.1038/nrd.2016.246

31. Schafer SA, Hulsewig C, Barth P, et al. Correlation between SFRP1 expression and clinicopathological parameters in patients with triple-negative breast cancer. Future Oncol. 2019;15 (16):1921-1938. doi:10.2217/fon-2018-0564

32. Regel I, Eichenmuller M, Mahajan UM, et al. Downregulation of SFRP1 is a protumorigenic event in hepatoblastoma and correlates with beta-catenin mutations. J Cancer Res Clin Oncol. 2020;146 (5):1153-1167. doi:10.1007/s00432-020-03182-1

33. Sunkara RR, Sarate RM, Setia P, et al. SFRP1 in skin tumor initiation and cancer stem cell regulation with potential implications in epithelial cancers. Stem Cell Reports. 2020;14(2):271-284. doi:10.1016/j. stemcr.2019.12.006
Cancer Management and Research

\section{Publish your work in this journal}

Cancer Management and Research is an international, peer-reviewed open access journal focusing on cancer research and the optimal use of preventative and integrated treatment interventions to achieve improved outcomes, enhanced survival and quality of life for the cancer patient.
The manuscript management system is completely online and includes a very quick and fair peer-review system, which is all easy to use. Visit http://www.dovepress.com/testimonials.php to read real quotes from published authors. 